\title{
Comparación del curso perinatal de las diferentes enfermedades hipertensivas del embarazo
}

Hernández-González Flavio*, Sandoval-Valdez Darío Alejandro*, Prieto-Macías Jorge**, TerronesSaldivar Ma. del Carmen***

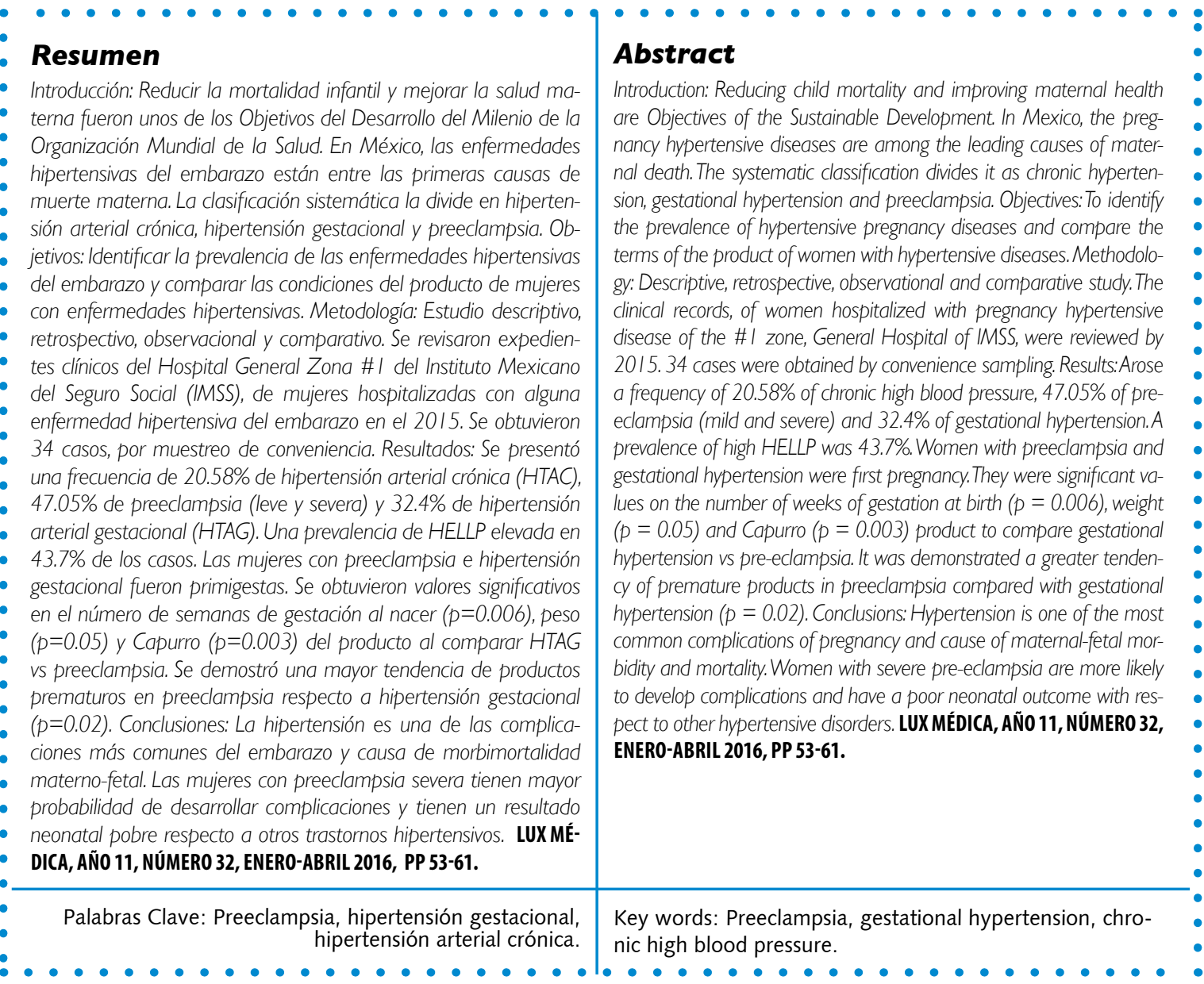

* Estudiantes del séptimo semestre de la carrera de Medicina del Centro de Ciencias de la Salud de la Universidad Autónoma de Aguascalientes.

** Médico Internista Cardiólogo. Maestro en Ciencias de la Educación. Jefe de la Unidad Médica Didáctica y Profesor investigador del Centro de Ciencias de la Salud de la Universidad Autónoma de Aguascalientes.

*** Ginecóloga, con Maestría en Ciencias y Doctorado en Farmacología. Profesora de tiempo completo e investigadora del Departamento de GinecoObstetricia del Centro de Ciencias de la Salud de la Universidad Autónoma de Aguascalientes

Fecha de recibido: 19 de enero 2016

Fecha de aceptación: 26 de febrero 2016

Correspondencia: Dra. en C. Ma. del Carmen Terrones Saldívar. Departamento de Gineco-Obstetricia y Pediatría. Edifico 107 planta alta. Ciudad Universitaria. Avenida Universidad 940. Código postal 20131, Aguascalientes, Ags., México. Teléfono 4499108443 Correo electrónico mcterron@correo.uaa.mx 


\section{Introducción}

La reducción de la mortalidad infantil y el mejoramiento de la salud materna fueron dos de los Objetivos del Desarrollo del Milenio de la Organización Mundial de la Salud (OMS) para el 2015, sin embargo, en nuestro país no se alcanzó a cumplir esta meta ${ }^{1}$. La tasa de mortalidad materna en las regiones en desarrollo es 14 veces mayor que en las regiones desarrolladas. Sólo la mitad de las mujeres embarazadas en regiones en desarrollo recibe la atención prenatal mínima recomendada de cuatro visitas ${ }^{2}$. En México, las enfermedades hipertensivas del embarazo se encuentran entre las primeras causas de muerte materna ${ }^{3}$. Debido a que es un grave problema de salud durante el embarazo, es trascendental la adecuada prevención, diagnóstico y tratamiento, todo esto para evitar un posible desenlace adverso. En general, se ha observado que las mujeres con preeclampsia obtienen productos con menor peso al nacer y de menor edad gestacional, y se han realizado varios estudios en grandes poblaciones que verifican esto ${ }^{4}$.

Los trastornos hipertensivos del embarazo se dividen en 4 grupos de acuerdo a la National High Blood Pressure Education Program Working Group (NHBPEP): ${ }^{5}$

- Hipertensión crónica o pre-existente: Hipertensión presente antes del embarazo o diagnosticada por primera vez antes de las 20 semanas de gestación.

- Hipertensión gestacional o hipertensión transitoria del embarazo: Hipertensión diagnosticada después de las 20 semanas, sin evidencia de proteinuria.

- Preeclampsia/eclampsia: Hipertensión diagnosticada de las 20 semanas de gestación con proteinuria o compromiso de algún órgano blanco.

- Preeclampsia sobreañadida a hipertensión crónica: Hipertensión presente antes del embarazo, en quien se incrementa los niveles de tensión arterial, se detecta proteinuria por primera vez o se agrava una ya existente.

Se ha reportado que aproximadamente $30 \%$ de las enfermedades hipertensivas asociados al embarazo que se diagnostican corresponden a hipertensión crónica o preexistente, mientras que el $70 \%$ son casos con diagnósticos de hipertensión gestacional/ preeclampsia ${ }^{6}$. La preeclampsia predispone hacia complicaciones potencialmente letales involucradas con el desprendimiento prematuro de placenta normoinserta, coagulación intravascular diseminada, hemorragia intracraneal, falla hepática, insuficiencia renal aguda y colapso cardiovascular. Crecimiento fetal intrauterino restringido, muerte fetal intrauterina y nacimiento prematuro son los problemas obstétricos relacionados ${ }^{7}$. La hipertensión arterial cró- 
nica o preexistente en el embarazo se define como aquella que se diagnosticó antes del embarazo o antes de la semana 20 de gestación y persiste después de las 6 semanas posparto, ocurre en $1 \%$ $5 \%$ de las mujeres embarazadas ${ }^{2}$. Se asocia a complicaciones como retardo en el crecimiento fetal, parto prematuro, insuficiencia renal aguda y crisis hipertensiva. El objetivo de este trabajo fue comparar los diferentes trastornos hipertensivos del embarazo y las principales complicaciones del binomio, así como estimar la frecuencia relativa de los diferentes trastornos hipertensivos del embarazo.

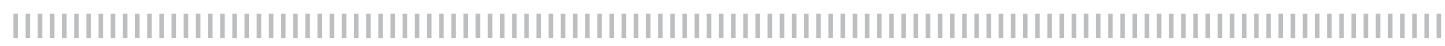

\section{Material y métodos}

Se realizó un estudio descriptivo, retrospectivo, observacional y comparativo. Se consideraron a las mujeres ingresadas de enero a septiembre de 2015 al Hospital General Zona \#1 IMSS con diagnóstico de enfermedad hipertensiva asociada al embarazo. Se realizó muestreo por conveniencia. Se registraron los antecedentes heredo-familiares, antecedentes personales patológicos, antecedentes ginecobstétricos, edad gestacional al momento de la resolución del embarazo, descripción de la resolución del embarazo y características del producto al nacimiento de cada paciente. Se revisaron los expedientes de pacientes con fecha de egreso hospitalario entre enero y septiembre de 2015 y con diagnóstico de enfermedad hipertensiva asociada al embarazo (Clasificación Internacional de Enfermedades (CIE) O10, O11, 013, O14 y 016). Se eliminaron aquellos casos que tuvieron el expediente incompleto.

Se realizaron 3 grupos según el trastorno hipertensivo del embarazo:
1) Hipertensión gestacional o hipertensión transitoria del embarazo

2) Hipertensión crónica o preexistente del embarazo

3) Preeclampsia / Preeclampsia sobreañadida a hipertensión crónica

Se definió como productos de bajo peso a aquellos con peso menor a 2500 gramos y como productos pretérmino a los que nacieron antes de las 37 semanas de gestación. Se utilizó estadística descriptiva para las características demográficas de cada grupo. Se realizaron pruebas no paramétricas (U de Mann-Whitney) para comparar las medias de semanas de gestación al nacimiento y peso del producto al nacer entre cada par de grupos. Se comparó entre los tres grupos usando la prueba no paramétrica de Kruskal-Wallis. Se utilizó la prueba de Chi-cuadrada para variables categóricas (proporción de productos pre término y proporción de productos de bajo peso) entre cada par de grupos. Se consideró significativo un valor de palor de $\mathrm{p}$ de dos colas $\leq 0.05$ e intervalos de confianza del $95 \%$.

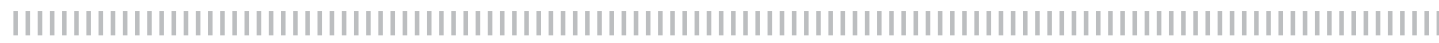

\section{Resultados}

Quedaron incluidas 34 mujeres que estuvieron internadas en el servicio de Ginecología y Obstetricia del Hospital General Zona \# 1 del IMSS del estado de Aguascalientes, con el diagnóstico de enfermedad hipertensiva asociada al embarazo.
La preeclampsia fue la complicación más frecuente, con el $47.05 \%$ de los casos estudiados, seguida de la hipertensión gestacional y de la hipertensión crónica, con un $32.4 \%$ y $20.5 \%$, respectivamente. La prevalencia de cada enfermedad hipertensiva se muestra en la gráfica 1. 


\section{Gráfica l}

Frecuencia de las enfermedades hipertensivas asociadas al embarazo: preeclampsia $(n=16)$, hipertensión gestacional (HTAG) $(n=11)$ e hipertensión crónica (HTAC) $(n=7)$.

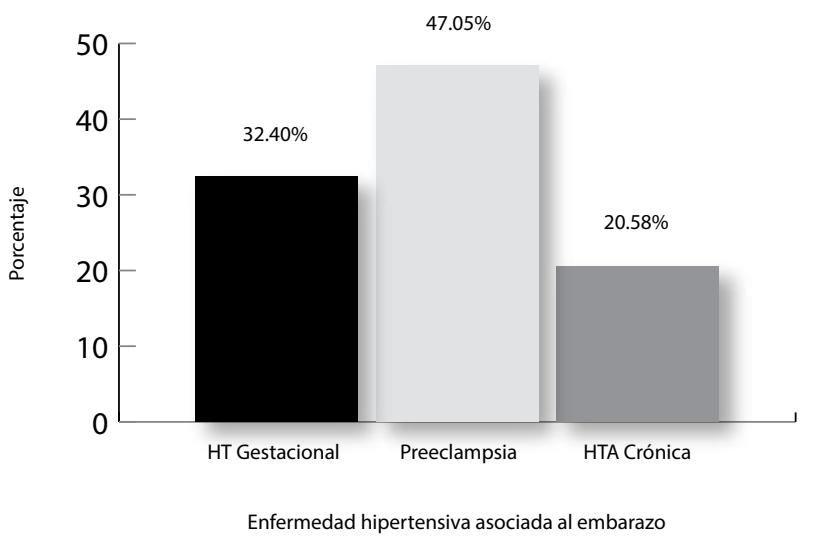

Hubo una prevalencia de síndrome de HELLP de $43.7 \% \quad(n=7)$ en el grupo de preeclampsia, el doble a la reportada en la bibliografía, en el grupo de HTAC en un $14.28 \%(n=1)$, mientras que en el grupo de HTAG no se reportó ningún caso, $8.82 \%(n=3)$ de la población total desarrollo oligohidramnios severo, sin observarse tendencias en cuanto a enfermedad hipertensiva, en $14.28 \%(n=1)$ se reportó muerte fetal tardía y $14.28 \%(n=1)$ desarrollo eclampsia.

Del total de mujeres, $61.76 \% \quad(n=21)$ afirmaron tener antecedentes heredo-familiares de diabetes mellitus, de igual manera $61.76 \% \quad(n=21)$ respondieron tener antecedentes familiares de hipertensión arterial, presentando las mujeres con hipertensión crónica un porcentaje mayor (85.7\%).

\section{Gráfica 2}

Distribución de las diferentes enfermedades hipertensivas asociadas a la gestación según su paridad.

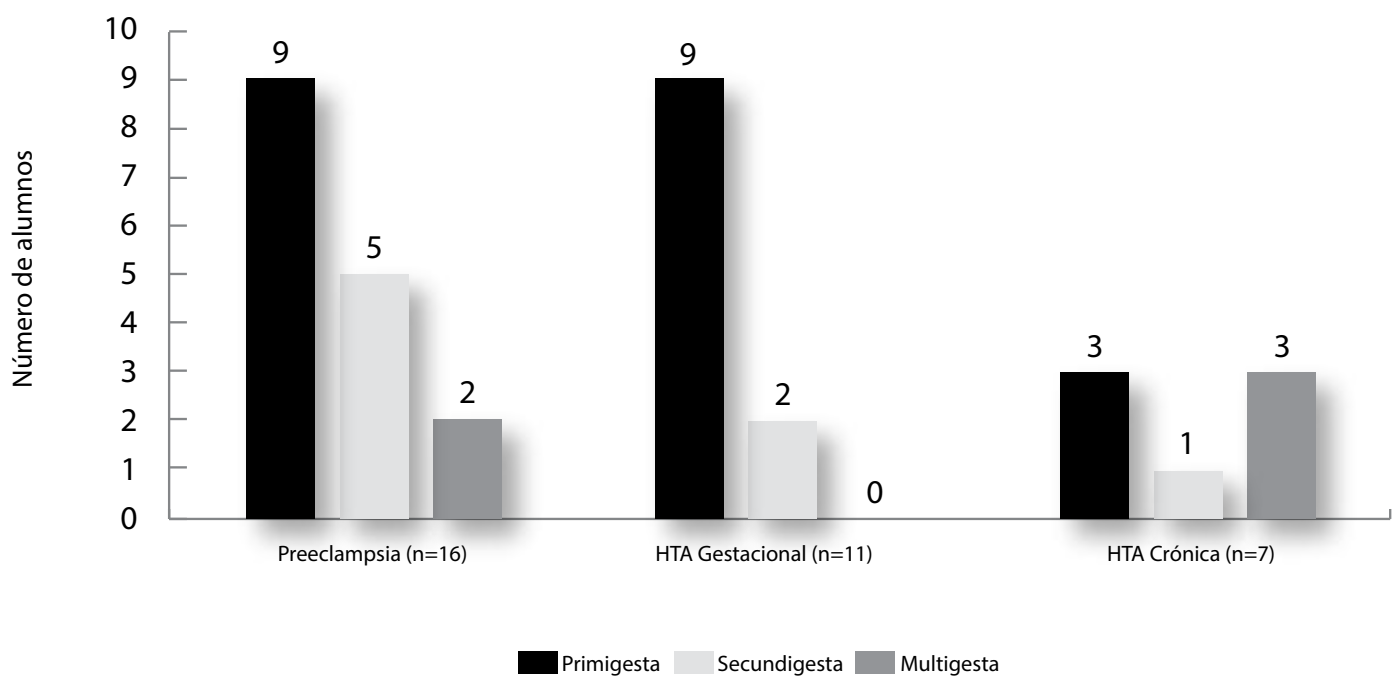


En la gráfica 2, se puede observar observar que la mayoría de las pacientes con preeclampsia e hipertensión gestacional fueron primigestas, mientras que en la paciente con hipertensión crónica hubo la misma cantidad de primigestas como de multigestas.

\section{Tabla I}

Resultados del cálculo de las medias \pm desviación estándar de edad de la madre y talla del producto, según el grupo de enfermedad hipertensiva asociada al embarazo.

\begin{tabular}{|c|c|c|c|}
\hline & $\begin{array}{l}\text { HTA crónica } \\
(n=6)\end{array}$ & $\begin{array}{l}\text { HTA gestacional } \\
(n=10)\end{array}$ & $\begin{array}{l}\text { Preeclampsia } \\
(n=15)\end{array}$ \\
\hline Edad (años) & $33.86 \pm 6.67$ & $29.18 \pm 6.66$ & $29.75 \pm 6.5$ \\
\hline Talla del producto $(\mathrm{cm})$ & $45 \pm 4.55$ & $49.5 \pm 1.84$ & $47.61 \pm 5.3$ \\
\hline
\end{tabular}

En la tabla 1 se puede observar que el grupo de HTAC fue el que registró una edad materna mayor (33.8 años \pm 6.6 años) que en el grupo de HTAG con (29.1 años \pm 6.6 años). La talla del producto fue menor en los neonatos de las mujeres del grupo de HTAC, con un promedio de 45 $\mathrm{cm} \pm 4.5 \mathrm{~cm}$.

Se excluyeron del cálculo de promedios de semanas de gestación (SDG) al nacer, peso y Capurro del producto a 3 pacientes, una mujer con diagnóstico de preeclampsia severa, por embarazo gemelar, que por condición propia los productos tienden a una talla baja. Una de hipertensión gestacional, por neonato de 6100 gramos y muerte neonatal temprana posteriormente, y una de hipertensión crónica, por óbito. De igual manera se dividió a la población en los tres grupos según su diagnóstico.

Se analizaron los datos con pruebas no paramétricas, U Mann-Whitney, al hacer comparaciones de hipertensión crónica vs hipertensión gestacional, preeclampsia vs hipertensión crónica e hipertensión gestacional vs preeclampsia se obtuvieron valores de $p$, como se muestran a continuación, en la tabla 2.

\section{Tabla 2}

Muestra las comparaciones de los parámetros: semanas de gestación al momento de nacer (SDG), peso y calificación de Capurro del producto, y número de gestas previas de la madre, y su respectivo valor $p$ tras pruebas $U$ Mann-Whitney. Hipertensión arterial (HTA). NV: Es no valorable por la prueba estadística al ser una población reducida.

\begin{tabular}{|c|c|c|c|c|c|c|}
\hline & $\begin{array}{l}\text { HTA crónica VS } \\
\text { HTA gestacional }\end{array}$ & $\mathbf{P}$ & $\begin{array}{l}\text { HTA crónica VS } \\
\text { Preeclampsia }\end{array}$ & $\mathbf{P}$ & $\begin{array}{l}\text { HTA gestacional } \\
\text { VS Preeclampsia }\end{array}$ & $\mathbf{P}$ \\
\hline SDG al nacer (semanas) & $\begin{array}{c}34.4+-5.24 \text { VS } \\
39.72+-1.65\end{array}$ & 0.01 & $\begin{array}{c}34.4+-5.24 \text { VS } \\
36.96+-2.4\end{array}$ & 0.5 & $\begin{array}{c}39.72+-1.65 \text { VS } \\
36.96+-2.4\end{array}$ & 0.006 \\
\hline Peso del producto (gramos) & $\begin{array}{c}2405+-719.03 \text { VS } \\
3321+-630.23\end{array}$ & 0.03 & $\begin{array}{l}2405+-719.03 \text { VS } \\
2635.71+-872.72\end{array}$ & 0.7 & $\begin{array}{l}3321+-630.23 \text { VS } \\
2635.71+-872.72\end{array}$ & 0.05 \\
\hline Capurro & $\begin{array}{c}36.05+-3.23 \text { VS } \\
39.23+-1.29\end{array}$ & NV & $\begin{array}{c}36.05+-3.23 \text { VS } \\
36.76+-2.01\end{array}$ & NV & $\begin{array}{c}39.23+-1.29 \text { VS } \\
36.76+-2.01\end{array}$ & 0.003 \\
\hline
\end{tabular}


La mayor cantidad de valores estadísticamente significativos se obtuvieron al comparar hipertensión gestacional vs preeclampsia. Los productos de las mujeres con preeclampsia tienen un promedio menor tanto en semanas de gestación al momento de nacer, siendo significativo con $(p=0.006)$, así como en peso y Capurro del producto, con valores significativos de $(p=0.05$ y $p=0.003)$, respectivamente. No hay diferencias significativas al comparar preeclampsia vs hipertensión crónica. Se obtuvieron valores estadísticamente significativos al comparar los productos de las mujeres con HTAC vs los de mujeres con HTAG, registrando un promedio mayor en los de hipertensión gestacional en SDG al nacer y peso del producto, siendo significativos estadísticamente con $(p=0.01 \mathrm{y}$ $p=0.03)$, respectivamente.

\section{Tabla 3}

Cálculo de prueba Chi-cuadrada de las variables categóricas de bajo peso y peso 2500 gr, en las poblaciones de preeclampsia vs HTA gestacional. $(p=0.08)$. Cálculo de prueba Chi-cuadrada de las variables de productos prematuros y productos > 37 SDG en las poblaciones de preeclampsia vs HTA gestacional. $(p=0.02)$

\begin{tabular}{|lcccc|}
\hline & Bajo Peso (<2500 gr) & Peso $>\mathbf{2 5 0 0}$ gr & Prematuros (<37 SDG) & >37 SDG \\
\hline Preeclampsia & $6(4.08)$ & $8(9.92)$ & $8(5.4)$ & $7(9.6)$ \\
\hline HTA gestacional & $1(2.92)$ & $9(7.08)$ & $1(3.6)$ & $9(6.4)$ \\
\hline
\end{tabular}

En la tabla 3 se obtuvo un valor de $p$ no significativo en la prueba de $2 \times 2$, para las características de bajo peso o peso $>2500$ gramos $(p=0.08)$. Pero sí fue significativa la comparación de prematuros y produc- tos mayores de 37 SDG $(p=0.02)$, lo cual demuestra una distribución de frecuencia mayor de productos prematuros, en el grupo de preeclampsia respecto al grupo de hipertensión gestacional.

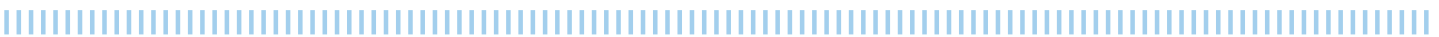

\section{Discusión}

Los desórdenes hipertensivos asociados al embarazo involucran un incremento de riesgos materno-fetales ${ }^{8}$. El médico debe identificar y diagnosticar oportunamente tales enfermedades, para así determinar la mejor estrategia de manejo médico-terapéutico, y por lo tanto disminuir las alteraciones materno-fetales.

Se asegura que la edad gestacional es la variable que funciona como el predictor más fuerte de morbimortalidad fetal, especialmente si es de menos de 30 semanas $^{7}$. En nuestra población tuvimos dos casos de productos con SDG $\leq 30$, ambos ca- sos pertenecían a madres con hipertensión crónica. En uno de los casos el producto fue óbito, mientras que el otro embarazo se complicó con preeclampsia severa y síndrome de HELLP, obteniendo un neonato de 1300 gr de peso. También se propone a la proteinuria, como el factor que incrementa el riesgo y la morbimortalidad materno-fetal ${ }^{9}$. Son diversas y numerosas las variables que determinan y contribuyen al pronóstico del binomio las enfermedades hipertensivas asociadas al embarazo.

En la bibliografía se reporta que las complicaciones fetales asociadas a la preeclampsia son crecimiento intrauterino 
restringido, oligohidramnios, producto pretérmino, puntuaciones de Apgar bajas ${ }^{10}$. Entre las complicaciones maternas reportadas durante el embarazo con alguna enfermedad hipertensiva son desprendimiento prematuro de placenta normoinserta, falla renal aguda, coagulación intravascular diseminada (CID), edema de pulmón, síndrome de distress respiratorio del adulto, desprendimiento de retina, evento vascular-cerebral (EVC) y muerte ${ }^{11}$. En nuestra población $8.82 \% \quad(n=3)$, una mujer de cada grupo, desarrolló oligohidramnios severo, lo cual complica el $0.7 \%$ de todos los embarazos $^{12}$. Está descrito que existen diversas condiciones adversas que incrementan el riesgo de complicaciones severas, y entre las que afectan la unidad feto-placentaria es el oligohidramnio ${ }^{13}$. No vimos diferencias significativas que pudiesen relacionar el desarrollo de esta complicación en un grupo en específico. Hubo un caso de EVC isquémico durante el puerperio en una mujer con hipertensión gestacional, se reporta en una prevalencia de $1.17 \%^{7}$, entretanto nuestra población mostró un $2.63 \%(n=1)$, duplicando la reportada. Se registró también un caso de falla renal aguda en una mujer con preeclampsia. La prevalencia de falla renal en mujeres embarazadas con trastornos hipertensivos es de $2.35 \%{ }^{7}$, en este estudio se registró una de $2.63 \%(n=1)$, similar a la reportada. Las complicaciones maternas generalmente se correlacionan con la severidad de la preeclampsia ${ }^{14}$, y ha sido demostrado que la falta de cuidados prenatales y Apgar $<5$ en el primer minuto está asociado con un pronóstico fetal pobre ${ }^{11}$. Las mujeres con preeclampsia registraron los niveles de tensión arterial mayores en nuestra población, sistólica de $164 \mathrm{mmHg} \pm 13 \mathrm{mmHg}$ y diastólica $99 \mathrm{mmHg} \pm 10 \mathrm{mmHg}$, en la bibliografía algunos meta-análisis las han promediado en $137.2 \mathrm{mmHg} \pm 12.5 \mathrm{mmHg}$ la sistólica y diastólica de $83.8 \mathrm{mmHg} \pm 11.9$ $\mathrm{mmHg}^{15}$.
Al contraponer HTAC vs preeclampsia, en las variables de SDG al nacer, número de gestas previas de la madre, el peso y el Capurro del producto no encontramos diferencias estadísticamente significativas. Por otro lado, se reporta que tanto las complicaciones maternas como las fetales están incrementadas en mujeres con el diagnóstico de hipertensión crónica ${ }^{7}$, otros estudios demuestran que dichas complicaciones son similares entre mujeres con preeclampsia leve e hipertensión gestacional, pero que existe un peor pronóstico en las mujeres con hipertensión crónica ${ }^{15}$.

Se ha comparado la hipertensión crónica leve, con hipertensión gestacional y con preeclampsia leve, concluyendo que las mujeres con hipertensión gestacional tienen los riesgos de morbilidad más altos respecto a las mujeres con enfermedades hipertensivas leves ${ }^{16}$. Los neonatos de mujeres con hipertensión gestacional y preeclampsia leve tienen mayor morbilidad comparados con los neonatos de mujeres con hipertensión crónica leve ${ }^{16}$. Lo que demostraría que las variaciones fisiológicas por más pequeñas que sean y que pueden llevar de enfermedad leve a severa, logran cambiar en gran medida el pronóstico del binomio.

Obtuvimos la mayor cantidad de valores estadísticamente significativos al comparar hipertensión gestacional vs preeclampsia. Los mejores promedios fueron los productos del grupo de mujeres con HTAG y los peores promedios los productos del grupo de preeclampsia, esto por la fisiopatología que representa la preeclampsia, que resulta de la alteración entre el suministro uteroplacentario y las demandas fetales, aunado a la activación endotelial y a la respuesta inflamatoria sistémica que conlle$v^{17}{ }^{17}$. Se documenta que el número elevado de mecanismos complejos que involucran la oxidación de lípidos y proteínas, la producción anormal de óxido nítrico y moléculas de adhesión, así como la expresión 
de glicoproteínas placentarias, juegan un papel importante en la disfunción trofoblasto-endotelio que podrían estar involucradas en la etiopatogenia y fisiopatología de la preeclampsia7. Los productos de las mujeres con preeclampsia tienden a un nacimiento prematuro, antes de 37 semanas, respecto a los productos de las mujeres con hipertensión gestacional $(p=0.02)$, si bien no obtuvimos valores significativos en la comparación de productos de peso bajo $(p=0.08)$, que podría estar explicado por la población pequeña o por las características de preeclampsia leve y preeclampsia severa unidas en el grupo.

En las comparaciones de SDG al momento de nacer, peso y Capurro del producto, las diferencias estadísticas se encontraron al comparar HTAG vs preeclampsia. Se observó que los productos de las mujeres con hipertensión gestacional tienen neonatos de mejor tamaño y de una edad gestacional mayor, ya que tendieron a nacer en una SDG mayor $(p=0.006)$, además de tener pesos y Capurros de mejor puntuación ( $p=0.05$ y $p=0.003$ ) respectivamente, mas no hay diferencias significativas al compararlos vs HTAC. Se obtuvieron valores estadísticamente significativos al comparar HTAC vs HTAG, tanto en SDG al nacer y peso $(p=0.01$ y $p=0.03)$, respectivamente.
No se observó ninguna diferencia significativa que pudiera asociar el ser primigestas o multigestas con desarrollar alguna enfermedad hipertensiva, quizás por tratarse de una población pequeña. Pero sí se observó una tendencia en las mujeres con preeclampsia $(n=9)$ y con hipertensión gestacional $(n=9)$, a ser primigestas. Se consideran como factores de riesgo para desarrollar preeclampsia la edad materna $\geq 40$ años, primiparidad o multiparidad, así como preeclampsia previa ${ }^{9}$, en nuestra población ninguna mujer fue mayor de 40 años, sólo una mujer del grupo de preeclampsia desarrolló preeclampsia en un embarazo previo.

Se ha sugerido que las mujeres con preeclampsia severa tienen una mayor probabilidad de desarrollar complicaciones maternas y tienen un resultado neonatal pobre ${ }^{4}$. En este trabajo se observó que los productos de las mujeres con hipertensión gestacional suelen tener mejores promedios en cuanto a semanas de gestación al nacer, lo que permite que se desarrollen mejor respecto a los productos de las mujeres con hipertensión crónica y preeclampsia. Los productos de las mujeres preeclámpticas tienden a tener productos de peso bajo, respecto a los de las mujeres con HTAG.

\section{Conclusiones}

La hipertensión es uno de las complicaciones más comunes del embarazo. La importancia de un manejo médico de cualquier tipo de las enfermedades hipertensivas asociadas al embarazo es la individualización de un plan terapéutico que debe asegurar un parto seguro y una buena salud fetal. Debido a que los resultados adversos durante el embarazo con algún trastorno hipertensivo son frecuentes, es necesario un énfasis en la estrategia de estudio y manejo de las mujeres con factores de riesgo, para mejorar la salud materno-fetal. 


\section{Bibliografía}

1. UN Millennium Project | Publications. Organización de las Naciones Unidas.

2. Guía de Práctica Clínica, Evidencias y Recomendaciones. Centro Nacional de Excelencia Tecnológica en Salud. México: Secretaría de Salud, 2010.

3. Numeralia 2013 - Observatorio de Mortalidad Materna.

4. Sibai BM. Diagnosis and management of gestational hypertension and preeclampsia. Obstet Gynecol. 2003 Jul; 102(1):181-92.

5.- Report of the National High Blood Pressure Education Program Working Group on High Blood Pressure in Pregnancy. Am J Obstet Gynecol. 2000 Jul;183(1):S1-22.

6. Ye C, Ruan Y, Zou L, Li G, Li C, Chen Y, et al. The 2011 Survey on Hypertensive Disorders of Pregnancy (HDP) in China: Prevalence, Risk Factors, Complications, Pregnancy and Perinatal Outcomes. PLoS One [Internet]. 2014 Jun 17 [cited 2015 Nov 13];9(6). Available from: http://www.ncbi.nlm.nih. gov/pmc/articles/PMC4061123/.

7. Yücesoy G, Ozkan S, Bodur H, Tan T, Cali?kan E, Vural $B$, et al. Maternal and perinatal outcome in pregnancies complicated with hypertensive disorder of pregnancy: a seven year experience of a tertiary care center. Arch Gynecol Obstet. 2005 Nov;273(1):439.

8. Cruz MO, Gao W, Hibbard JU. Obstetrical and perinatal outcomes among women with gestational hypertension, mild preeclampsia, and mild chronic hypertension. Am J Obstet Gynecol. 2011 Sep;205(3):260.e1-9.

9. Fatimah Soydemir LK. Hypertension in pregnancy. Current Obstetrics \&amp; Gynaecology. 2006;16(6):315-20.

10. Magee LA, Pels A, Helewa M, Rey E, von Dadelszen $P$, Canadian Hypertensive Disorders of Pregnancy (HDP) Working Group, et al. The hypertensive disorders of pregnancy (29.3). Best Pract Res Clin Obstet Gynaecol. 2015 Jul;29(5):643-57.

11. Liu C-M, Cheng P-J, Chang S-D. Maternal complications and perinatal outcomes associated with gestational hypertension and severe preeclampsia in Taiwanese women. J Formos Med Assoc. 2008
Feb;107(2):129-38.

12. Nabhan AF, Abdelmoula $Y A$. Amniotic fluid index versus single deepest vertical pocket as a screening test for preventing adverse pregnancy outcome. Cochrane Database Syst Rev. 2008;(3):CD006593.

13. Magee LA, Pels A, Helewa M, Rey E, von Dadelszen $P$, SOGC Hypertension Guideline Committee. Diagnosis, evaluation, and management of the hypertensive disorders of pregnancy: executive summary. J Obstet Gynaecol Can. 2014 Jul;36(7):575-6.

14. Sibai B, Lecarpentier E, Tsatsaris V, Goffinet F, Cabrol D, Haddad B (2013) Risk Factors of Superimposed Preeclampsia in Women with Essential Chronic Hypertension Treated before Pregnancy. PLoS ONE 8(5): e62140

15. Bramham K, Parnell B, Nelson-Piercy C, Seed PT, Poston L, Chappell LC. Chronic hypertension and pregnancy outcomes: systematic review and metaanalysis. BMJ. 2014;348:g2301.

16. van Oostwaard MF, Langenveld J, Schuit E, Papatsonis DNM, Brown MA, Byaruhanga RN, et al. Recurrence of hypertensive disorders of pregnancy: an individual patient data metaanalysis. Am J Obstet Gynecol. 2015 May;212(5):624.e1-17.

17. Turner JA. Diagnosis and management of preeclampsia: an update. Int J Womens Health. 2010;2:327-337.

18. Payne BA, Groen H, Ukah UV, Ansermino JM, Bhutta Z, Grobman W, et al. Development and internal validation of a multivariable model to predict perinatal death in pregnancy hypertension. Pregnancy Hypertens. 2015 Oct;5(4):315-321.

19. Terrone DA, Rinehart BK, May WL, Moore A, Magann EF, Martin JN. Leukocytosis is proportional to HELLP syndrome severity: evidence for an inflammatory form of preeclampsia. South Med J. 2000 Aug;93(8):768-771.

20. Magee LA, von Dadelszen $P$, Rey E, Ross $S$, Asztalos $E$, Murphy KE, et al. Less-tight versus tight control of hypertension in pregnancy. N Engl J Med. 2015 Jan 29;372(5):407-417.

21. Matthys LA, Coppage KH, Lambers DS, Barton JR, Sibai BM. Delayed postpartum preeclampsia: an experience of 151 cases. Am J Obstet Gynecol. 2004 May;190(5):1464-1466. 